\title{
Seroprevalencia de la enfermedad de Chagas en el cantón Aguarico, Amazonía ecuatoriana
}

\author{
Manuel Amunárriz, ${ }^{1}$ Segundo Quito, ${ }^{2}$ Víctor Tandazo ${ }^{2}$ y Mayra López ${ }^{2}$
}

Forma de citar Amunárriz M, Quito S, Tandazo V, López M. Seroprevalencia de la enfermedad de Chagas en el cantón Aguarico, Amazonía ecuatoriana. Rev Panam Salud Publica. 2010;28(1):25-9.

RESUMEN Objetivo. Determinar el estado actual de la seroprevalencia de la enfermedad de Chagas en la población del cantón Aguarico, y comparar los resultados obtenidos con datos provenientes de otros estudios epidemiológicos.

Métodos. Desde septiembre de 2008 hasta octubre de 2009 se recogieron 2033 sueros correspondientes a 36,6\% de la población total del cantón Aguarico. Se empleó la técnica CHAGATEST/ELISA recombinante v.3.0 para determinar positividad a la enfermedad de Chagas. Los casos positivos se confirmaron con pruebas de hemoaglutinación indirecta y ELISA.

Resultados. De las 2033 muestras de suero obtenidas, 73 fueron positivas para Chagas $(3,6 \%)$. El rango de variación de seroprevalencia en las comunidades con casos positivos osciló entre 1,4\% y 13,3\%. Un 60,3\% de los casos positivos se encontraron en el sexo femenino. Estos hallazgos son similares a los de estudios previos de comparación realizados en 1990.

Conclusiones. Los datos obtenidos confirman la existencia de un foco autóctono de la enfermedad de Chagas en la Amazonía ecuatoriana, con un porcentaje superior a la media de la región amazónica. No se detectaron cuadros clínicos agudos ni patologías crónicas. Es perentorio implementar un programa de control de la enfermedad culturalmente adaptado a la región.

Palabras clave Enfermedad de Chagas; comunidades indígenas; salud indígena; estudios seroepidemiológicos; ecosistema amazónico; Ecuador.

En los países de la región amazónica -Bolivia, Brasil, Colombia, Ecuador, Guayana Francesa, Guyana, Perú, Suriname y Venezuela- la enfermedad de Chagas fue considerada irrelevante hasta aproximadamente los años 1980. Desde esa época, diversos estudios han dado a conocer tanto casos agudos como con serologías positivas en habitantes amazónicos de casi todos los países mencionados (1). Luego, a partir de 2002, se realizaron numerosos encuentros auspiciados por organismos internacionales ${ }^{3}$

\footnotetext{
1 Hospital Franklin Tello, Nuevo Rocafuerte, Orellana, Ecuador. Facultad de Ciencias de la Salud Eugenio Espejo, Universidad Tecnológica Equinoccial (UTE), Quito, Ecuador. La correspondencia debe dirigirse a Manuel Amunárriz, mamunarrizu01@yahoo.es

2 Hospital Franklin Tello, Nuevo Rocafuerte, Orellana, Ecuador.
}

para establecer políticas de control de la enfermedad e investigación de los mecanismos de transmisión del vector $(2,3)$. Hoy en día nadie duda de que la enfermedad de Chagas sea una patología autóctona e importante en la región Amazónica, sujeta a mecanismos biológicos y ambientales de transformación, y que requiere esfuerzos especiales tanto en investigación como en atención sanitaria.

En la Amazonía ecuatoriana, los primeros datos sobre esta enfermedad corresponden a pacientes con cuadros agudos recopilados entre 1987 y 1992, en la

\footnotetext{
Entre ellos, la Red Latinoamericana de Investigación sobre la Biología y el Control de Triatominos (ECLAT, por su sigla en inglés), en 2002, y la Iniciativa Intergubernamental de Vigilancia y Prevención de la Enfermedad de Chagas en la Amazonía (AMCHA), en 2004, 2005 y 2006.
}

región nororiental. Los 12 pacientes fueron localizados en las actuales provincias de Orellana y Sucumbíos, y el diagnóstico se realizó por el hallazgo de Trypanosoma cruzi en sangre periférica. La mayoría de los casos fueron diagnosticados y tratados en el Hospital Franklin Tello de Nuevo Rocafuerte, cantón Aguarico. En esa época, y en diversas zonas amazónicas, se identificaron tres especies de triatominos infestados con formas epimastigotes y tripomastigotes de T. cruzi: Panstrongylus geniculatus, Rhodnius pictipes y Rhodnius robustus (4-6).

En 1990 se llevó a cabo una investigación sobre seroprevalencia de la enfermedad de Chagas en las comunidades indígenas del río Napo, entre Rocafuerte y Coca, en la misma región nororiental. Se tomaron sueros de 1011 habitantes, se 
congelaron en nitrógeno líquido y se enviaron a Quito, donde fueron sometidos a la prueba de microELISA para Chagas con antígeno crudo. Los resultados de este estudio fueron comunicados de manera preliminar en 1994 y posteriormente, tras un análisis más exhaustivo, en $1997(6,7)$.

A partir de entonces el tema de la incidencia de Chagas en las poblaciones nativas de la Amazonía ha sido investigado en varias ocasiones, concretamente en $2003(8,9)$ y 2007 (1). Uno de los estudios de 2003, realizado en 6866 muestras recogidas en 162 comunidades, arrojó una tasa de seroprevalencia de $2,4 \%$, resultado que sugería una transmisión activa de Chagas en la región amazónica ecuatoriana asociada a las pobres condiciones de las viviendas (9). Dada la necesidad de actualizar los datos y facilitar las medidas de control, se creyó oportuno realizar un nuevo estudio transversal de seroprevalencia de Chagas en un área más reducida y mejor delimitada de la Amazonía ecuatoriana, situada cerca de la confluencia de los ríos Napo y Aguarico, en el cantón Aguarico, provincia de Orellana. El presente trabajo tiene precisamente el objetivo de determinar la seroprevalencia de la enfermedad de Chagas en la población del cantón Aguarico en 2008-2009 y comparar esos resultados con datos provenientes de otros estudios epidemiológicos.

\section{MATERIALES Y MÉTODOS}

Toda la población estudiada, de etnia fundamentalmente indígena, reside en plena selva amazónica, dispersa en las márgenes de los ríos Napo y Aguarico. Nuevo Rocafuerte, la cabecera cantonal, tiene 516 habitantes, la mayoría mestizos asentados desde principios del siglo XX. Tiputini, con unos 700 habitantes, es la segunda población en importancia administrativa del cantón y sede de un comando militar con funciones de control fronterizo. El resto del cantón está organizado en comunas, cada una con su centro comunal y su escuela primaria. Las viviendas, a orillas del río, están construidas con material de selva y abiertas a la naturaleza exuberante que las rodea. Sus encuentros comunitarios, si no frecuentes, sí son periódicos, y se relacionan con los compromisos participativos en la vida de la comunidad.

El área estudiada está formada por seis parroquias o circunscripciones civi- les que agrupan a 29 comunidades indígenas, con una población total estimada de 5550 habitantes. Y si bien el territorio escogido es más pequeño que los correspondientes a los estudios de 1990 y 2003, la muestra estudiada representa un porcentaje mayor de la población total.

El estudio utilizó un diseño transversal. En diferentes visitas, realizadas entre septiembre de 2008 y octubre de 2009, se recogió una muestra no aleatoria de 2033 sueros que corresponden a 36,6\% de la población total, provenientes de miembros que residen en 27 de las 29 comunidades de la zona de estudio. Dada la naturaleza e idiosincrasia de la población indígena, el número de sueros obtenidos estuvo basado en la aportación voluntaria de la población que acudía a los centros comunales para una asistencia médica general. El muestreo incluyó a personas de todas las edades, desde niños hasta adultos mayores. Las repetidas visitas a las comunidades indígenas permitieron una labor de concientización sobre la naturaleza y la gravedad de la enfermedad de Chagas, lo que hizo que la colaboración de la población creciera a lo largo del período de toma de muestras.

El tamaño de la muestra estuvo basado en la tasa de seroprevalencia obtenida en el estudio de 1990 (6,03\%), con un intervalo de confianza de $99 \%$ y un resultado mínimo aceptable de $4 \%$. Debido a la selección no aleatoria de la muestra, se tuvo en cuenta un efecto de diseño de 2. El cálculo realizado con el programa Epi Info dio un total de 1568 muestras.

Las pruebas serológicas se realizaron en el laboratorio del Hospital Franklin Tello, empleando el método ELISA con la técnica CHAGATEST/ELISA recombinante v.3.0. Se trata de un ensayo inmunoenzimático (ELISA) de $3^{\mathrm{a}}$ generación para la detección de anticuerpos contra el T. cruzi, elaborado por WIENER Laboratorios SAIC (Rosario, Argentina). La lectura de resultados se realizó con el equipo STAT FAX 303/PLUS, de Awareness Technology, Inc. (Florida, Estados Unidos). Se consideraron positivos aquellos sueros con absorbancia por encima de 1300 . La longitud de onda primaria empleada para la lectura fue de $450 \mathrm{~nm}$, y la de onda secundaria (dicromática), de 620-650 nm.

Las muestras seropositivas fueron enviadas al Instituto Nacional de Higiene de
Guayaquil para pruebas inmunológicas de validación. En esa institución, sobre cada muestra se realizaron dos pruebas: una de hemoaglutinación indirecta (HAI) y una de ELISA. Todos los sueros positivos, menos dos, fueron confirmados por ambas pruebas. Todos los casos positivos fueron sometidos a un detallado examen clínico y un electrocardiograma.

\section{RESULTADOS}

De las 2033 muestras de suero obtenidas en el presente estudio, $73(3,6 \%)$ fueron positivas para Chagas. Hubo diferencias importantes en cuanto al porcentaje de positividad entre las diversas comunidades, con variaciones que oscilaron entre 1,4\% y 13,3\%. En 9 de las 27 comunidades estudiadas hubo más de $7 \%$ de resultados positivos (cuadro 1).

La distribución por sexo de la población examinada fue equilibrada, con $53,3 \%$ de mujeres. Los casos positivos fueron considerablemente superiores en el sexo femenino, con $60,3 \%$ frente a $39,7 \%$ para el masculino. La distribución etaria de los casos positivos muestra algunos datos a tener en cuenta, comenzando con el grupo de 0 a 5 años, que registró una prevalencia baja $(0,8 \%)$. En los grupos siguientes los resultados positivos se hicieron progresivamente más frecuentes, manteniendo una prevalencia superior a $6 \%$ a partir de los 20 años, excepto en el grupo de 30 a 39 años $(2,4 \%)$. Se observó asimismo una aparente reducción general, y por grupos de edad, de la prevalencia en relación a los datos de 1990 (cuadro 2).

Del total de la muestra, 37 mujeres estaban embarazadas al momento de la toma del suero, dos de las cuales dieron positivo para Chagas (5,4\%); tiempo después, los dos bebés fueron sometidos a la prueba de ELISA: un niño de cinco meses, con resultado negativo, y una niña de dos meses, que dio positivo.

En ninguna de las personas con serología positiva se detectó, en el examen clínico, sintomatología cardiaca o digestiva ni alteraciones en el electrocardiograma.

\section{DISCUSIÓN}

En un lapso de aproximadamente 20 años (1987-2009) se han realizado tres estudios epidemiológicos sobre la incidencia de la enfermedad de Chagas en la Amazonía ecuatoriana: AmunárrizChico (1990), Grijalva (2003) $(5,9)$ y el 
CUADRO 1. Comparación de los resultados de tres estudios realizados en la Amazonía ecuatoriana para determinar la seroprevalencia de la enfermedad de Chagas, Ecuador, 2009a

\begin{tabular}{|c|c|c|c|c|c|c|c|}
\hline \multirow[b]{2}{*}{ Comunidad } & \multirow{2}{*}{$\begin{array}{c}\begin{array}{c}\text { Estudio } \\
\text { de } 1990^{b}\end{array} \\
\%\end{array}$} & \multicolumn{3}{|c|}{ Estudio de 2003} & \multicolumn{3}{|c|}{$\begin{array}{l}\text { Estudio de Aguarico } \\
\qquad(2008-2009)\end{array}$} \\
\hline & & Muestras & Positivas & $\%$ & Muestras & Positivas & $\%$ \\
\hline Fronteras de Ecuador & $N D^{c}$ & 39 & 5 & 12,8 & 42 & 4 & 9,5 \\
\hline Martinica & 5,6 & 59 & 1 & 1,7 & 34 & 3 & 8,8 \\
\hline Nuevo Rocafuerte & 3,2 & 310 & 11 & 3,5 & 342 & 5 & 1,5 \\
\hline Belo Horizonte & ND & ND & ND & ND & 13 & 1 & 7,7 \\
\hline Santa Teresita & ND & 189 & 7 & 3,7 & 73 & 1 & 1,4 \\
\hline Alto Florencia & ND & ND & ND & ND & 48 & 1 & 2,1 \\
\hline Santa Rosa & ND & 138 & 6 & 4,3 & 39 & 1 & 2,6 \\
\hline Puerto Miranda & ND & 45 & 4 & 8,9 & 43 & 1 & 2,3 \\
\hline Pandochicta & ND & 40 & 2 & 5,0 & 61 & 5 & 8,2 \\
\hline Llanchama & ND & ND & ND & ND & 48 & 1 & 2,1 \\
\hline Boca Tiputini & ND & ND & ND & ND & 67 & 2 & 3,0 \\
\hline San Carlos & ND & ND & ND & ND & 64 & 3 & 4,7 \\
\hline Tiputini & 0,0 & 250 & 2 & 0,8 & 266 & 5 & 1,9 \\
\hline Vicente Salazar & ND & ND & ND & ND & 11 & 1 & 9,1 \\
\hline Puerto Quinche & 5,8 & 84 & 2 & 2,4 & 191 & 9 & 4,7 \\
\hline Huiridima & 0,0 & 44 & 4 & 9,1 & 55 & 3 & 5,4 \\
\hline San Vicente & 1,6 & 139 & 9 & 6,5 & 94 & 4 & 4,2 \\
\hline Centro Ocaya & ND & ND & ND & ND & 37 & 4 & 10,8 \\
\hline Sinchichicta & ND & 69 & 9 & 13,0 & 59 & 1 & 1,7 \\
\hline Chiruisla & 7,7 & 175 & 9 & 5,1 & 136 & 4 & 2,9 \\
\hline Limonyacu & ND & 38 & 3 & 7,9 & 38 & 4 & 10,5 \\
\hline Nueva Esperanza & ND & ND & ND & ND & 15 & 2 & 13,3 \\
\hline Samona & ND & 37 & 2 & 5,4 & 69 & 2 & 2,9 \\
\hline Moretecocha & ND & ND & ND & ND & 21 & 1 & 4,8 \\
\hline Chonta Urcu & ND & ND & ND & ND & 11 & 1 & 9,1 \\
\hline Zancudo & ND & ND & ND & ND & 73 & 0 & 0 \\
\hline Sabalo & ND & ND & ND & ND & 7 & 0 & 0 \\
\hline PI Cuyabeno & ND & ND & ND & ND & 37 & 2 & 5,4 \\
\hline Kawimeno & ND & ND & ND & ND & 39 & 2 & 5,1 \\
\hline Total & 3,4 & 1656 & 76 & 4,6 & 2033 & 73 & 3,6 \\
\hline
\end{tabular}

a Los estudios de referencia son: en 1990, Amunárriz-Chico (5), en 2003, Grijalva y colaboradores (9) y, en 2008-2009, Amunárriz y colaboradores (presente trabajo). Las pruebas serológicas de los estudios de 1990 y de 2008-2009 fueron realizadas en el Hospital Franklin Tello, Nuevo Rocafuerte, Orellana.

${ }^{b}$ En este estudio no fue posible acceder a datos segmentados por comunidades, por lo que no se presenta información sobre el tamaño de la muestra y el número de casos positivos.

${ }^{c} \mathrm{ND}=$ No disponible.

CUADRO 2. Resultados comparados de dos estudios de seroprevalencia para enfermedad de Chagas, por grupos de edad, Ecuador, 2009

\begin{tabular}{|c|c|c|c|c|c|c|}
\hline \multirow{2}{*}{$\begin{array}{l}\text { Grupo } \\
\text { etario } \\
\text { (años) }\end{array}$} & \multicolumn{3}{|c|}{ Estudio de 1990 (río Napo)a $^{a}$} & \multicolumn{3}{|c|}{ Estudio de Aguarico (2008-2009) } \\
\hline & Examinados & Positivos & $\begin{array}{c}\text { Prevalencia } \\
(\%)\end{array}$ & Examinados & Positivos & $\begin{array}{c}\text { Prevalencia } \\
(\%)\end{array}$ \\
\hline $1-5$ & 42 & 2 & 4,8 & 117 & 1 & 0,8 \\
\hline $6-10$ & 130 & 2 & 1,5 & 627 & 7 & 1,1 \\
\hline $11-19$ & 272 & 12 & 4,4 & 544 & 23 & 4,2 \\
\hline $20-29$ & 215 & 6 & 2,8 & 266 & 18 & 6,8 \\
\hline $30-39$ & 148 & 7 & 4,7 & 168 & 4 & 2,4 \\
\hline $40-49$ & 101 & 13 & 12,9 & 138 & 9 & 6,5 \\
\hline $50-59$ & 69 & 13 & 18,8 & 94 & 6 & 6,4 \\
\hline$\geq 60$ & 34 & 6 & 17,6 & 79 & 5 & 6,3 \\
\hline Totales & 1011 & 61 & 6,0 & 2033 & 73 & 3,6 \\
\hline
\end{tabular}

a Incluye las comunidades indígenas establecidas a lo largo del río Napo, desde Nuevo Rocafuerte hasta Coca (5).

b Comprende comunidades indígenas situadas dentro del cantón Aguarico.

presente (2008-2009). Estos trabajos permiten confirmar algunos datos relevantes $y$, al mismo tiempo, plantean preguntas que deberán ser abordadas en investigaciones posteriores que profun- dicen el conocimiento de esta importante patología parasitaria. Los tres estudios, no obstante, corroboran la presencia de una patología chagásica autóctona en la Amazonía de Ecuador.
En 1978-1991, los 12 casos agudos conocidos correspondían, en su mayoría (83\%), a pacientes colonos asentados en la región desde hacía largo tiempo. La naturaleza de estos casos indicaba la adquisición reciente de la enfermedad $y$, por lo tanto, que era originaria del lugar y no importada de otra zona. Por esa misma época, se notificaron dos casos agudos de niños indígenas pertenecientes a comunidades de la ribera del Napo, cuya relación con un posible origen importado hay que descartar. Los estudios posteriores, destinados a determinar la seroprevalencia para Chagas en las comunidades del río Napo, han confirmado la existencia de casos positivos en las comunidades indígenas de todas las áreas habitadas de la vasta región amazónica ecuatoriana.

Por otra parte, a partir de 1989, en diferentes áreas de los cantones Orellana y Aguarico, y muy separadas unas de otras, se localizaron tres especies de triatominos: Panstrongylus geniculatus, Rhodnius pictipes y Rhodnius robustus. Ejemplares de las tres especies estaban parasitados por formas epimastigotes y tripomastigotes de $T$. cruzi, confirmadas mediante examen directo de heces en el laboratorio del Hospital Franklin Tello. Es importante constatar la amplia distribución de estos vectores en la Amazonía y su grado de parasitación (10-13). Al parecer, la parasitación de los triatominos existentes en la región mantiene un ciclo biológico de infestación permanente con mamíferos amazónicos parasitados como reservorios naturales de $T$. cruzi. Los casos humanos de parasitación solo pueden explicarse como situaciones esporádicas de esta zoonosis.

La prevalencia total observada en el presente estudio es prácticamente la mitad $(3,6 \%)$ de la encontrada en el trabajo de 1990, que abarcó los cantones de Aguarico y Orellana. Sin embargo, si los datos de 1990 se circunscriben a las comunidades del cantón Aguarico, la prevalencia es de 3,4\%, muy similar a la obtenida en el presente trabajo.

El estudio de 1990, realizado en población indígena (1 011 muestras, seroprevalencia total de 6,03\%), encontró una prevalencia de $4,8 \%$ en el grupo etario de 1 a 5 años, aumentando progresivamente a partir de los 20 años. Una progresión similar fue hallada en el presente estudio. El aumento de la prevalencia en los grupos de mayor edad podría explicarse por el tiempo en que dicha población ha estado expuesta al contacto con los vec- 
tores de la enfermedad. No está muy claro, sin embargo, por qué en este estudio la prevalencia en el grupo de edad de 30 a 39 años disminuye.

El trabajo de 2003 incluyó comunidades de cuatro provincias amazónicas: Napo, Orellana, Pastaza y Sucumbíos. Orellana, donde se encuentra el cantón Aguarico, presentó una prevalencia de $3,4 \%$, con $1,2 \%$ en el grupo de edad de cero a 10 años (7). Ambos resultados son similares a los del presente trabajo (cuadro 2).

La evaluación del estado clínico de los pacientes y de los datos sobre su historia clínica realizada en este estudio dio lugar a dos observaciones que se repiten continuamente. La primera es la inexistencia, salvo en un único caso, de cuadros agudos al comienzo de la instalación de la parasitosis por T. cruzi. Aunque numerosas personas refirieron haber sido picadas por chinches y la aparición de una o varias reacciones locales dermocutáneas (chagomas) que duraron algún tiempo, en ningún caso mencionaron síntomas clínicos. Parece poco probable la existencia de otras vías de entrada del parásito, como por ejemplo la oral, si tenemos en cuenta las características biológicas extradomiciliarias del vector y las costumbres alimentarias de la población $(14,15)$. A pesar de esto, sería relevante investigar en profundidad este aspecto de la biología del vector.

La segunda observación recurrente es la inexistencia de pacientes con sintomatología cardiaca y alteraciones en el electrocardiograma entre los casos positivos de la muestra examinada. De igual manera, el examen clínico descartó síntomas sugerentes de alteraciones del tracto digestivo, como megaesófago o megacolon. Se reconocen, no obstante, las limitaciones del examen clínico practicado y la necesidad de investigaciones más profundas para descartar estas patologías.

En la actualidad, si bien hay consenso sobre la conveniencia de tratar los casos humanos de la enfermedad de Chagas, aún aquellos considerados como Chagas indeterminados, no lo hay en cuanto a la valoración de su eficacia ni a los criterios que deben adoptarse para evaluar su efectividad $(16,17)$. De acuerdo con la Dirección Nacional Ecuatoriana de Control de la enfermedad de Chagas, en una segunda etapa de investigación, se proyecta someter a la población seropositiva a un tratamiento con Nifurtimox y medir el grado de efectividad de este medicamento.

También es necesario señalar algunas limitaciones del presente estudio. Una se refiere a las técnicas que se emplearon para obtener la muestra. La configuración de las comunidades del cantón Aguarico se caracteriza por una gran dispersión de la población, lo que dificultó considerablemente la obtención de una muestra aleatoria; al mismo tiempo, la participación voluntaria en la muestra podría estar sobreestimando la prevalencia encontrada. Por otro lado, en la investigación realizada no se abordó el tema del vector. Las especies existentes, el porcentaje de infestación en la población de triatominos y el carácter extra o intradomiciliario, están basados en información obtenida durante el período 1987-1992. Hará falta entonces una actualización de esos datos que permita saber más acerca de las vías de infección humana.

Un tercer aspecto que se debe tener en cuenta es el hecho de carecer de datos completos de las investigaciones realizadas en los años 1990 y 2003, lo cual ha limitado la precisión de las comparaciones. Los diferentes métodos diagnósticos utilizados en distintos laboratorios podrían contribuir a variar las prevalencias obtenidas. Lamentablemente, tampoco se pudieron realizar pruebas cruzadas con leishmaniasis. No obstante, el haber obtenido una prevalencia similar a la de 1990, refuerza los hallazgos actuales.

En cuanto a la muestra positiva de Chagas encontrada en un recién nacido, se debe esperar un plazo razonable, no inferior a seis meses, para repetir la prueba y asegurar que su inmunorreacción obedece a una parasitación verdadera por vía transplacentaria y no al paso de los anticuerpos elaborados por su madre. Al momento de escribir este artículo, dicha prueba todavía no se había podido realizar.

En conclusión, puede afirmarse que la enfermedad de Chagas no es importada desde regiones de la costa ecuatoriana, a través de procesos de migración poblacional, sino que se trata de una enfermedad autóctona de la región amazónica, originada por la existencia de una verdadera zoonosis mantenida por ciclos biológicos propios del lugar. Debido a factores que por el momento no se pueden determinar, la prevalencia de la infección humana por T. cruzi en el cantón Aguarico es mayor que la media de la región amazónica, estimada entre $1 \%$ y $2 \%$. Los casos agudos son escasos y los crónicos, con sintomatología cardiaca o digestiva, excepcionales. La mayoría de los casos podrían clasificarse como "Chagas indeterminados".

El conjunto de investigaciones realizadas en la Amazonía ecuatoriana en los 20 últimos años permite insistir en algunas recomendaciones para el futuro próximo. La primera es la necesidad de abordar el estudio de triatominos para determinar con mayor precisión las especies existentes y su nivel de infestación y, sobre todo, constatar si en algunas circunstancias se da un proceso de domiciliación en sus hábitos de vida. En segundo lugar, hace falta comprobar, en un programa de terapia sobre Chagas, el grado de eficacia de los antichagásicos utilizados y las pautas concretas que se deben emplear en una región como la amazónica. Finalmente, y englobando las recomendaciones anteriores, es perentorio implementar un programa culturalmente adaptado de control de la enfermedad de Chagas en la Amazonía de Ecuador.

\section{REFERENCIAS}

1. Aguilar HM, Abad-Franch F, Pinto Dias JC, Veríssimo Junqueira AC, Rodrigues Cousa J. Chagas disease in the Amazon region. Mem Inst Oswaldo Cruz. 2007;102(1 supl):47-55

2. Organización Panamericana de la Salud (OPS). AMCHA: iniciativa de los países amazónicos para la prevención y control de la enfermedad de Chagas. Washington DC: OPS; 2004.

3. Organización Panamericana de la Salud. Conclusiones y recomendaciones de la reunión anual conjunta IPA-AMCHA (Quito, Ecuador, 18- 20 septiembre 2006). Hallado en: http://www.paho.org/Spanish/AD/DPC/
CD/dch-amcha-ipa-2006.pdf. Acceso el 18 de junio de 2010.

4. Amunárriz M. Enfermedad de Chagas; primer foco amazónico. En: Estudios sobre patologías tropicales en la Amazonía ecuatoriana. Pompeya, Ecuador: CICAME; 1991. Pp. 27-36. 
5. Amunárriz M, Chico ME, Guderian RH. Chagas disease in Ecuador: a sylvatic focus in the Amazon Region. J Trop Med Hyg. 1991;94: $145-9$.

6. Amunárriz M. Enfermedad de Chagas. En: Crónicas incompletas sobre patologías tropicales desde la selva amazónica. Quito, Ecuador: CICAME; 1994. Pp. 75-87.

7. Chico HM, Sandoval C, Guevara EA, Calvopiña HM, Cooper JPh, Reed SG, et al. Chagas disease in Ecuador: Evidence for disease transmission in an indigenous population in the Amazon region. Mem Inst Oswaldo Cruz. 1997;92(3):317-20.

8. Abad-Franch F, Aguilar HM. Control de la enfermedad de Chagas en el Ecuador. Quito: OPS/MPS; 2003.

9. Grijalva MJ, Escalante L, Paredes RA, Costales JA, Padilla A, Rowland EC, et al. Seroprevalence and risk factors for Tripanosoma cruzi infection in the Amazon region of Ecuador. Am J Med Hyg. 2003;69(4):380-5.
10. Valente SA, da Costa Valente V, das Neves Pinto AY, de Jesus Barbosa César M, dos Santos MP, Miranda CO, et al. Analysis of acute Chagas disease outbreak in the Brazilian Amazon: human cases, triatomines, reservoir mammals and parasites. Trans $\mathrm{R}$ Soc Trop Med Hyg. 2009;103(3):291-7.

11. Guhl F, Aguilera G, Pinto N, Vargara D. Distribución de las especies de triatominos en los departamentos endémicos para la enfermedad de Chagas en Colombia. Primer taller internacional sobre control de la enfermedad de Chagas (2-6 de mayo de 2005). Bogotá, Colombia; 2005.

12. Valente VC, Valente SAS, Noireau F, Carrasco HJ, Miles MA. Chagas disease in the Amazon basin: association of Panstrongylus geniculatus (Hemiptera: Reduviidae) with domestic pigs. J Med Entomol. 1998;35:99-103.

13. Carcavallo RU, Galíndez Girón I, Jurberg J Lent $\mathrm{H}$. Atlas of Chagas disease vectors in the Americas. Rio de Janeiro: Fiocruz; 1997.
14. Nóbrega AA, Garcia MH, Tatto R, Obara MT, Costa E, Sobel J, et al. Oral transmission of Chagas disease by consumption of açai palm fruit, Brazil. Emerg Infect Dis. 2009;15(4): 653-5.

15. Pan American Health Organization (PAHO) Informe de la Consulta Técnica en Epidemiología, Prevención y Manejo de la Transmisión de la Enfermedad de Chagas como Enfermedad Transmitida por Alimentos (ETA). Río de Janeiro, Brasil; 2006.

16. Apt W. Tratamiento de la enfermedad de Chagas. Parasitol día. 1999;23(3-4).

17. Pinto Dias JC. Tratamiento etiológico de la enfermedad de Chagas: una síntesis. XIIa Reunión Intergubernamental INCOSUR/Chagas. Santiago de Chile, marzo 2003.

Manuscrito recibido el 14 de enero de 2010. Aceptado para publicación, tras revisión, el 9 de junio de 2010.

ABSTRACT Objective. Determine the current seroprevalence of Chagas disease in the population of Aguarico canton, and compare the findings with data from other epidemiological studies.

\section{Seroprevalence of Chagas disease in Aguarico canton in the Ecuadorian Amazon}

Key words
Methods. From September 2008 to October 2009, 2033 serum samples were collected from $36.6 \%$ of the total population in Aguarico canton. The Chagatest recombinant ELISA v.3.0 was used to determine positivity to Chagas disease. Cases that tested positive were confirmed by indirect hemagglutination and ELISA.

Results. Of the 2033 serum samples obtained, 73 tested positive for Chagas disease (3.6\%). Seroprevalence in communities with positive cases ranged from $1.4 \%$ to $13.3 \%$, with $60.3 \%$ of the positive cases found in females. These findings are similar to the 1990 comparison studies.

Conclusions. The data obtained confirm the existence of an indigenous focus of Chagas disease in the Ecuadorian Amazon, with a percentage higher that the average for the Amazon region. No acute clinical cases or chronic pathologies were detected. Implementation of a culturally appropriate Chagas control program for the region is urgently needed.

Chagas disease; indigenous population; indigenous health; seroepidemiological studies; Amazonian ecosystem; Ecuador. 